American Journal of Animal and Veterinary Sciences 5 (3): 196-201, 2010

ISSN 1557-4555

(C) 2010 Science Publications

\title{
Ultrastructural Changes in the Ileum of White BALB/C Mice Experimentally Infected with Salmonella hadar
}

\author{
Afaf Abdulrahman Yousif and Maan M. Neima AL-Naqeeb \\ Department of Internal and Preventive Medicine, College of Veterinary Medicine, \\ Ministry of Higher Education and Scientific Research, University of Baghdad, \\ Baghdad Al Karih-Al-Ameria, College of Veterinary Medicine, \\ Post Office Al-Dawoodi, P.O. Box, 28601 Iraq
}

\begin{abstract}
Problem statement: Recently, Salmonella hadar has been isolated and identified from goat in Iraq. The purpose of the present study was to investigate the ultrastructural changes in the ileum epithelial cells of mice experimentally infected with Salmonella hadar. Approach: The white BALB/c mice inoculated orally with Salmonella enterica serovar hadar strain and their ileums were examined by transmission electron microscopy at 24, 48, 72, 96 and $120 \mathrm{~h}$ after inoculation. Results: The challenge organism appeared adhered to the mucosal microvilli of the ileum from $24 \mathrm{~h}$ postinoculation. The early ultrastructural changes characterized by local derangement with slight swelling of the proximal ends of the microvilli. In addition, there was ruffling of the apical cell surfaces of the microvilli with cytoplasmic vacuolization of enterocytes due to invading by the organisms. The Challenge organism was usually intact and enclosed by a membrane. The damage of the epithelial cells started as a minor lesion at $24 \mathrm{~h}$ and became severe after 48, 72 and $96 \mathrm{~h}$ post infection. The more severe ultrastructural changes in the ileum occurred at $120 \mathrm{~h}$ post infection, which revealed hypertrophy of goblet cell, dilatation of endoplasmic reticulum, severe cytoplasmic vacuolization, thickening of the nuclear membrane and several Salmonella Containing Vacuoles (SCV). Conclusion: Our results revealed that the cellular invasion by Salmonella hadar may occurred early than $24 \mathrm{~h}$ because of the obvious alterations seen in the cells of the ileum at this time of infection. In addition, the continuation of cellular pathological changes for $120 \mathrm{~h}$ post infection may refers that $S$. hadar has some mechanisms which aid it to survive and replicate within intestinal cells. We recommend further studies with early observation periods in order to determine the invasion time.
\end{abstract}

Key words: Cytoplasmic vacuolization, electron microscopy, Salmonella hadar, ultrastructural

\section{INTRODUCTION}

Salmonella species are facultative intracellular gram-negative bacteria that cause a wide array of disease including systemic disease and enterocolitis in a multitude of hosts (Antunes et al., 2003). Murine infection with Salmonella enteric serovar typhimurium has been used predominantly to model human typhoid (caused by Salmonella enterica serovar Typhi), while bovine infection with Salmonella enterica serovar Dublin or S. enterica serovar typhimurium has been a prevailing model of intestinal disease. These models have been exploited to gain critical insight into the pathogenesis of disease cause by Salmonellae, including (for example) that invasion-associated genes are required for intestinal secretory and inflammatory disease, that intracellular survival in both the intestinal epithelium and macrophages is essential for systemic pathogenesis and that M cells of the ileal Peyer's patches are the site of invasion for systemic infection in murine typhoid prior to dissemination to liver and spleen via the reticuloendothelial system (Naughton et al., 2001; Poppe, 1999; Rahman et al., 2000; Wehkamp et al., 2004).

During recent years, Salmonella enterica subspecies enterica serovar hadar (Salmonella hadar) has been isolated almost worldwide at increasing frequencies not only from cases of clinical infections in human but also from those of food poisoning and from animals (Fantasia et al., 1997; Di Giannatale et al., 2008).

The first significant interactions between the bacteria and the host occur at the lymphoid follicles of

Corresponding Author: Afaf Abdulrahman Yousif, Department of Internal and Preventive Medicine, College of Veterinary Medicine, Ministry of Higher Education and Scientific Research, University of Baghdad, Baghdad Al Karih-Al-America, Post Office Al-Dawoodi, P.O. Box 28601, Iraq 
the intestine. This action would seem to play into the hands of the host because the primary function of lymphoid follicles is to protect the host from intestinal invasion. However, Salmonella displays a highly evolved ability not only to initiate contact with the host at Peyer's patches but to benefit from the interaction. Subsequently, the bacteria move into the lymphatic system where the survival of the organisms requires the ability to productively interact with professional killing cells. Again, these pathogens display a remarkable adaption to an environment that is considered to be very hostile. Thus, it is apparent that the bacteria have discovered that survival within the host depends on their ability to directly engage the host immune system and alter the host response in their favor. It seems certain that the pressure being exerted by this and other bacterial pathogens is honing the host response to infection. It is also clear that the bacteria will not be left behind in the evolutionary battle. From a scientific point of view, these interactions offer the opportunity to gain insights both into cell biology and bacterial pathogenicity (MacDonald and Monteleone, 2005; Santos et al., 2003).

Both Scanning and Transmission Electron Microscopes (SEM and TEM) have been used widely in the study of intestinal colonization by Salmonellae (Soliman et al., 2009; Ohl and Miller, 2001). The ultrastructural study had been done previously (YashRoy, 2007), by other researchers they explained the invasion mechanisms of a human isolate Salmonella in the chicken ileum, in addition they showed that over $70 \%$ of Salmonellae interacting with ileal epithelial cells developed numerous surface blebs of periplasmic extensions designated periplasmic organelles. The invading organisms enter the lymphatic vessels, where they again appeared to be closely interacting with ileal macrophages. Inside the cytoplasm of macrophages, numerous tight phagosomes were seen, each containing two organisms. While the final stage appeared to contain replicated Salmonellae and at the same time, macrophages also showed signs of apoptotic disintegration, culminating in the release of replicated Salmonellae.

Recently, Salmonella hadar has been isolated from goat in Iraq, to our knowledge; no previous study has been done to study the pathological change at the ultrastructural level, so the present study was designed to investigate the ultrastructural changes of the mice ileal epithelial cells experimentally infected with Salmonella hadar.

\section{MATERIALS AND METHODS}

Bacterial strain: Salmonella hadar was isolated from Iraqi goats, diagnosed and confirmed according to
(Quinn et al., 1993) and serotyped in the national salmonella center in Iraq. Estimating the bacterial Infectious Dose (ID) Five colonies of $S$. hadar inoculated in $10 \mathrm{~mL}$ of brain heart infusion broth at $37^{\circ} \mathrm{C}$ for $18 \mathrm{~h}$, then centrifuged in cold centrifuge at $8000 \mathrm{rpm}$ (round per min) for $15 \mathrm{~min}$ then the sediment washed three times with PBS $(\mathrm{pH}=7.2)$ and re-suspended with $1 \mathrm{~mL}$ of PBS ( $\mathrm{pH}=7.2)$, then tenfold dilution $\left(10^{-1}, 10^{-2}, 10^{-3}\right.$, $10^{-4}, 10^{-5}, 10^{-6}, 10^{-7}, 10^{-8}, 10^{-9}$ and $10^{-10}$ ) were done from the viable count of the bacteria. Each diluents were made according to method of Miles et al. (1938) and the following concentrations were selected: $\left(1.5 \times 10^{5}\right.$ cells $)$, $\left(1.5 \times 10^{6}\right.$ cells $),\left(1.5 \times 10^{7}\right.$ cells $),\left(1.5 \times 10^{8}\right.$ cells $),\left(1.5 \times 10^{9}\right.$ cells $),\left(1.5 \times 10^{10}\right.$ cells $)$ and $\left(1.5 \times 10^{11}\right.$ cells $)$. Seven groups of mice drenched orally with one of the calculated Colony Forming Unit (CFU. mL) diluted with $1 \mathrm{ml}$ PBS while the last group served as control and drenched with $1 \mathrm{ml}$ PBS $(\mathrm{pH}=7.2)$. All groups were observed for 30 days to calculate the live and dead mice, the Infective Dose (ID) was estimated by choosing the group of mice which showed clinical signs resemble to those in Salmonellosis with no mortality after ensure that by culture. This dose was calculated according to method described previously (Blaser and Newman, 1982).

Inoculation of laboratory animals: Forty mice (BALB/c), (6-8) weeks old of both genders were adapted for two weeks before starting the experiment. The mice were reared in separated clean and disinfected cages and fed on ad libitum commercial assorted pellets and clean water. Then, the mice divided into 2 groups each group with twenty mice. First group was the infected group which inoculated orally with $1.5 \times 10^{7}$ cells of Salmonella hadar suspended in $1 \mathrm{~mL}$ of PBS and the second group was control group which was drenched orally with phosphate buffer saline $(\mathrm{pH}=7.2)$. Four mice of each group were anesthetized at intervals 24, 48, 72, 96 and $120 \mathrm{~h}$ post infection and a part of small intestine (ileum) from both groups was taken for transmission electron microscope investigation.

Transmission Electron Microscope (TEM): Mice were anesthetized by diethyl ether at the specified periods by inhalation the ileum was removed quickly and drained. For transmission electron microscopy study, $1 \mathrm{~mm}$ portion of each ileum was taken and fixed in $2.5 \%$ gluteraldehyde and post-fixed in aqueous $1 \%$ osmium tetroxide $\left(\mathrm{OsO}_{4}\right)$ and in spur resin. After examination of the survey semi-ultrathin sections, ultrathin sections were cut and stained with uranyl acetate followed by lead citrate. The specimen was examined by transmission electron microscope Philips (CM-10). 


\section{RESULTS}

The ileum of all mice in the control group killed at different interval stages, revealed no abnormalities in the ultrastructural features and showed brush border of the columnar epithelial cells that covering the villi which consisted of long closely packed regular microvilli and normal cytoplasmic region (Fig. 1) However, the ileum of the infected groups showed several ultrasturactural. The ultrastructural features that especially characterized the experimental infection are, the fate of the salmonella organisms, certain responses of the intestinal epithelium to the bacterial invasion and the reaction of mucosal vessels to bacterial penetration into the lamina propria. The ileum of infected mice killed at $24 \mathrm{~h}$ post infection revealed different ultrastructural changes.

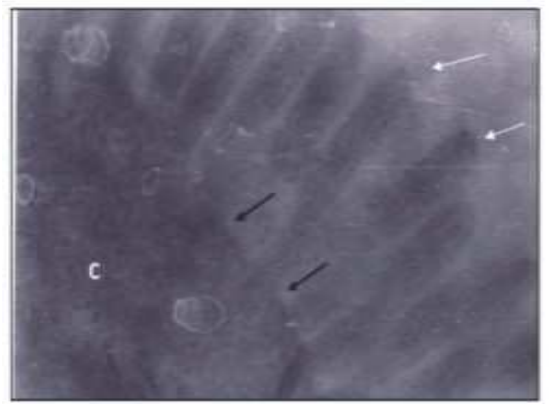

Fig. 1:Transmission electron micrograph. Ileum, mouse of control group, shows long, closely packed regular microvilli (white arrow), normal brush border (black arrow) with no changed in the cytoplasm of the Enterocyte (C). (Uranyl acetate and Lead citrate $) \times 64000$

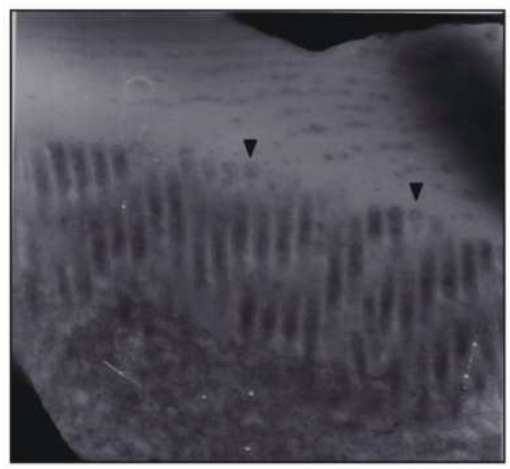

Fig. 2:TEM. Ileum, mouse (infected group) at $24 \mathrm{~h}$ post infection with Salmonella hadar, shows ballooning formation on the distal end of the microvilli (black head arrow). (Uranyl acetate and Lead citrate $) \times 31000$
The bacteria were found in the lumen of ileum as well as attached to the surface and associated with morphological alteration in the brush border of the enterocytes, which include the damaged of the brush border and local derangement with mild swelling particularly on the distal end of the microvillus that give the cells a ballooning appearance (Fig. 2 and 3).

The ultrastructural changes became severe at 48 and $72 \mathrm{~h}$ post infection and characterized by the injury of the microvillus and showed partial or complete detached. In addition there were some abnormalities of the cytoplasmic matrix involving vacuolization and marked dilatation of endoplasmic reticulum (Fig. 4).

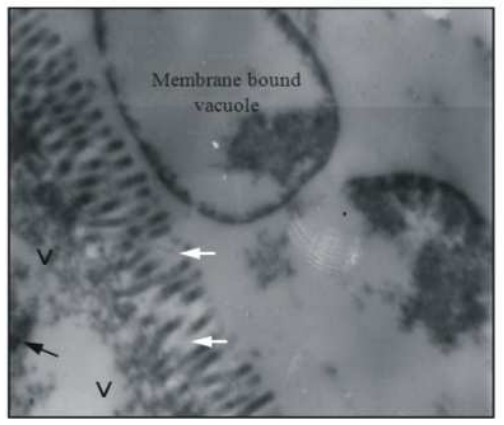

Fig. 3: TEM. Ileum, mouse (infected group) at $24 \mathrm{~h}$ post infection with $S$. hadar, shows bacterial attachment and invasion led to detachment of microvilli (white arrow), the microvilli and some microorganism are breakup in the membrane bound fragment, damage of the epithelial brush border and cytoplasm (v) also notice the presence of intracellular bacterium (black arrow). (Uranyl acetate and Lead citrate) $\times 16000$

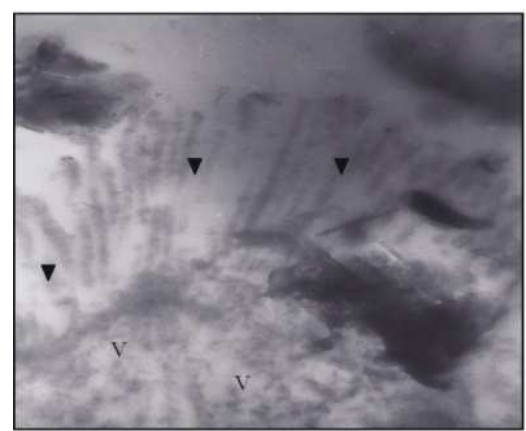

Fig. 4:TEM. Ileum, mouse (infected group) at $48 \mathrm{~h}$ post infection with $S$. hadar, Shows Complete damages to the microvilli (black head arrow) with cytoplasmic Vacuolation (V). (Uranyl acetate and Lead citrate) $\times 31000$ 


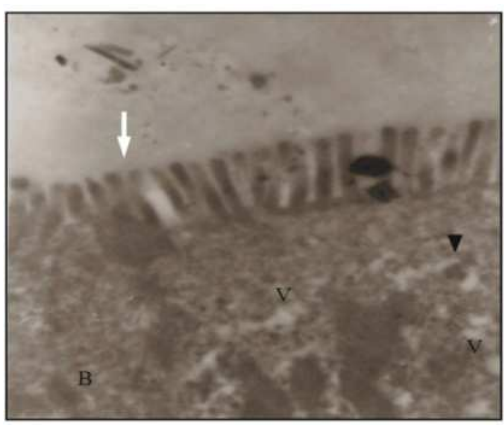

Fig. 5: TEM. Ileum, mouse (infected group) $72 \mathrm{~h}$ post infection with $S$. hadar, shows there is disproportional of the microvilli (white arrow), invading Bacteria (B), cytoplasmic Vacuolization (V) and cytoplasmic vacuole containing Salmonella (black head arrow). (Uranyl acetate and Lead citrate) $\times 16000$

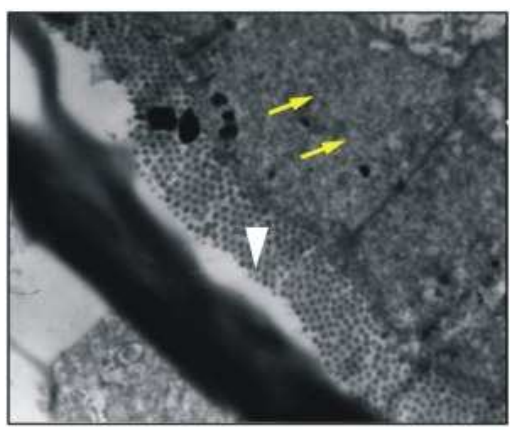

Fig. 6: TEM. Ileum, mouse (infected group ) $72 \mathrm{~h}$ post infection with $S$. hadar, shows disorganization of the M-cells (yellow arrow) with presence of Salmonella containing vacuole SCV (white head arrow) and invading salmonella (white arrow). (Uranyl acetate and Lead citrate) $\times 8700$

The cellular changes were more pronounced at $72 \mathrm{~h}$ (Fig. 5 and 6). The lesions and the damage became easily noticeable in the ileum at $96 \mathrm{~h}$ post infection and characterized by loss of some microvillus, marked dilatation and vacuolization of the endoplasmic reticulum with dispersion of microvillus and loss of the other mainly structures of the injured enterocytes due to presence of many intracellular bacteria (Fig. 7-9). The ileum of mice that killed at 120 post infection $h$ revealed the more severe ultrastructural changes including the hypertrophy of goblet cell, dilatation of endoplasmic reticulum, severe cytoplasmic vacuolization, thickening of the nuclear membrane and there were several Salmonella Containing Vacuoles (SCV) (Fig. 10 and 11).

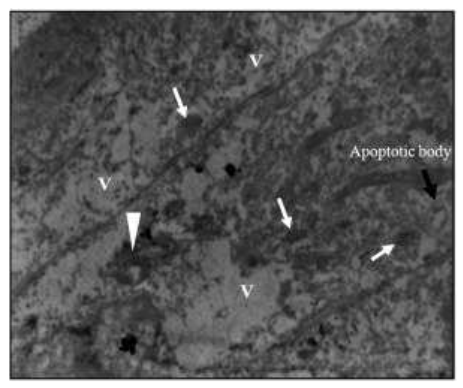

Fig. 7: TEM. Ileum, mouse (infected group) at $96 \mathrm{~h}$ post infection with $S$. hadar, shows marked dilatation of the endoplasmic reticulum (white head arrow) with vacuolation of the cytoplasm of the enterocytes (V), presence many intracellular bacteria (white arrow) also notice the apoptotic body (black arrow) $\times 4400$

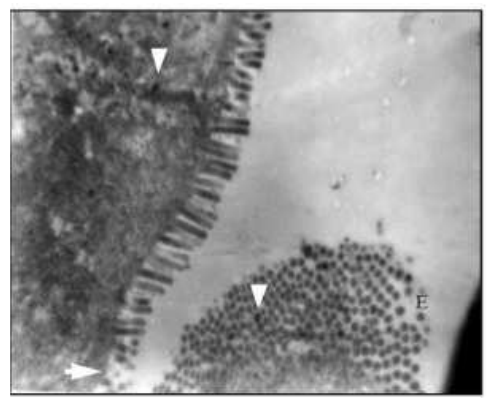

Fig. 8: TEM. Ileum, mouse (infected group) at $96 \mathrm{~h}$ post infection with $S$. hadar, shows loss of some microvilli (white arrow), presence of intracellular Salmonella in both an enterocytes and M-cell (white head arrow) with damage to the site of entry in M-cell (E). (Uranyl acetate and Lead citrate $) \times 12500$

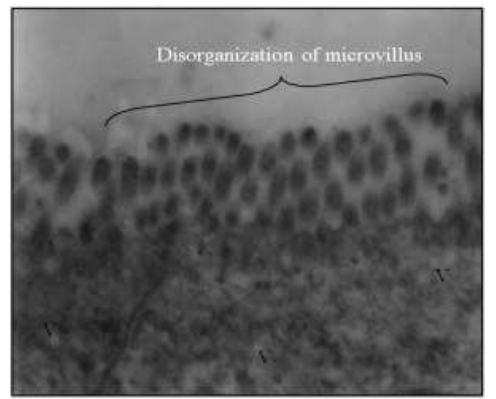

Fig. 9: TEM. Ileum, mouse (infected group) at 96 hours post infection with $S$. hadar, shows disorganization of the microvilli with Vacuolation spaces (V). (Uranyl acetate and lead citrate $) \times 31000$ 


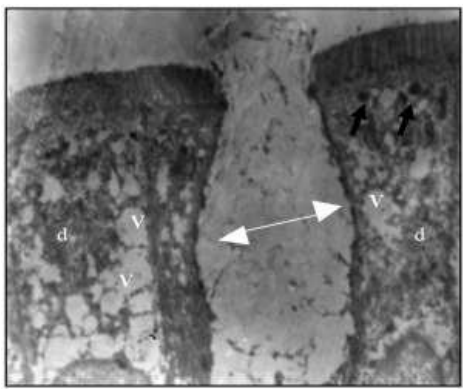

Fig. 10: TEM. Ileum, mouse (infected group) at $120 \mathrm{~h}$ post infection with $S$. hadar, shows hypertrophy of goblet cell (double head arrow), dilatation of endoplasmic reticulum (d), severe cytoplasmicvacuolization $(\mathrm{V})$ and presence of many intracellular bacteria (black arrow). (Uranyl acetate and Lead citrate) $\times 3400$

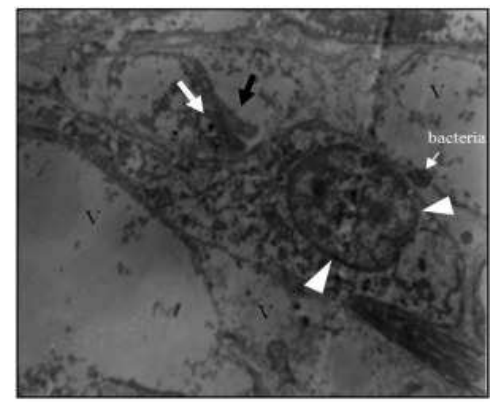

Fig. 11:TEM. Ileum, mouse (infected group) at $120 \mathrm{~h}$ post infection with $S$. hadar, shows dilatation of the endoplasmic reticulum (black arrow), increases in the thickening of the nuclear membrane (white head arrow), severe vacuolization of the enterocytes (V) with presence of $S$. hadar containing vacuole (white arrow) (Uranyl acetate and Lead citrate) $\times 3400$

\section{DISCUSSION}

The early ultrastructural changes in the infected group mice with $S$. hadar revealed that the microvilli of the ileum characterized by local derangement with little swelling on the proximal end. The ballooning of the microvilli which had been seen in the ileum at the ultrastructural level is one of the interesting findings which support the early attachment of $S$. hadar to the mucous membrane. While, when the infection was became more advanced, the microvilli became short and more effacement from epithelial cells in some location, these ultrastructural changes in the microvilli were similar to the ultrastructural changes reported previously by other researchers (Worton et al., 1989), they studied the ultrastructural changes of the microvilli of ileum in the rabbit experimentally infected with $S$. typhimurium. The effacement of the brush border of the enterocytes of the microvilli experimentally infected by $S$. hadar resulted in the loss of brush border enzymes (amino oligo peptidase, aspartate amino peptidase, dipeptidyl amino peptidase, carboxy peptidase and $\gamma$ glutamyl transferase) which hydrolyze small peptides that are formed in the intestinal lumen by the action of proteases to free amino acids during the process of absorption, carrier protein and surface area as mentions previously (Tobey et al., 1985). The shortening and loss of microvillus is in accordance with decreasing of alkaline phosphatase activity and this enzyme is located in the plasma membrane of the microvilli and is considered as a measurement of the digestiveabsorptive surface (Cote et al., 2004). The fragmentation and vesiculation of microvilli may be due to increases in cytoplasmic $\mathrm{Ca}^{2+}$ concentration hich lead to the actin filaments of the microvillus core cytoskeleton to be broken into short filament as mentioned previously (Embaye et al., 1989). The ultrastructural changes was observed in the epithelial cells were probably due to the presence of many intracellular Salmonella that produced cytotoxin inside the cells, these observations is similar to that observed previously in the small intestinem of the rat infected experimentally with $S$. typhimurium (Naughton et al., 1996) and also with that found in the ileum of swine which infected with $S$. typhimurium DT104 (Meyerholz et al., 2002). The loss of microvilli reduced the intestinal absorption surface, this together with the functional deficiency and destruction of many epithelial cells, lead to decrease in the absorption and caused gastroenteritis and diarrhea. The ultrastructural changes observed in the cytoplasm of the enterocytes which manifested by vacuolization of cytoplasm, displacement of organelles and swollen mitochondria, these changes in the host cells were occurred as a result of the effect of $S$. hadar endotoxin that is part of the outer membrane or metabolic substances released from the bacteria as mentioned previously (Ohl and Miller, 2001).

\section{CONCLUSION}

The conclusion of this study is the ileum of White BALB/C mice experimentally infected with Salmonella hadar revealed different ultrastructural change. These changes are different from a minor lesion at $24 \mathrm{~h}$ characterized by local derangement and slight swelling of the proximal ends of the microvilli with ruffling of the apical cell surfaces associated with cytoplasmic vacuolization of enterocytes due to invading by the organisms, to severe lesion at 48, 72 and $96 \mathrm{~h}$ post infection and the more sever ultrastructural changes, which occurred at $120 \mathrm{~h}$ post infection, which revealed hypertrophy of goblet cell, dilatation of endoplasmic 
reticulum, severe cytoplasmic vacuolization, thickening of the nuclear membrane and several Salmonella Containing Vacuoles (SCV). The challenge bacteria were seen at all post infection stages usually intact and enclosed by a membrane. We conclude that these obvious ultrastuctural finding approve that cellular invasion by Salmonella hadar probably occurred early than $24 \mathrm{~h}$ and the severity of the ultrastructural changes at $120 \mathrm{~h}$ post infection may refers that $S$. hadar has another mechanisms that aid their pathogeniciy to survive and replicating within the intestinal cells.

\section{REFERENCES}

Antunes, P., C. Reu, J.C. Sousa, L. Peixe and N. Pestana, 2003. Incidence of Salmonella from poultry products and their susceptibility to antimicrobial agents. Int. J. Food Microbiol., 82: 97-103. DOI: $10.1016 / \mathrm{S} 0168-1605(02) 00251-9$

Blaser, M.J. and L.S. Newman, 1982. A review of human salmonellosis: I. Infective dose. Rev. Infect. Dis., 4: 1096-1106. PMID: 6760337

Cote, S., A. Letellier, L. Lessard and S. Quessy, 2004. Distribution of Salmonella in tissues following natural and experimental infection in pigs. Can. J. Vet. Res., 68: 241-248. PMID: 15581217

Di Giannatale, E., V. Prencipe, V. Acciarri, M.M. Marconi and P. Semprini et al., 2008. Investigation of an outbreak of Salmonella enterica subsp. enterica serovar Hadar food illness in the Abruzzi region of Italy. Vet. Ital., 44: 417-427. PMID: 20405442

Embaye, H., R.M. Batt, J.R. Saunders, B. Getty and C.A. Hart, 1989. Interaction of enteropathogenic Escherichia coli $\mathrm{O} 111$ with rabbit intestinal mucosa in vitro. Gastroenterology, 96:1079-1086. PMID: 2647575

Fantasia, M., B. Paglietti, E. Filetici, M.P. Anastasio and S. Rubino, 1997. Conventional and molecular approaches to isolates of $S$. hadar from sporadic and epidemic cases. J. Applied Microbiol., 82: 494-498. DOI: 10.1046/j.1365-2672.1997.00142.x

MacDonald, T.T. and G. Monteleone, 2005. Immunity, inflammation and allergy in the gut. Science, 307: 1920-1925. DOI: 10.1126/science.1106442

Meyerholz, D.K., T.J. Stebel, M.R. Ackermann, S.A. Carlson and B.D. Jones et al., 2002. Early epithelial invasion by Salmonella enterica serovar typhimurium DT104 in the swine ileum. Vet. Pathol., 39: 712-720. PMID: 12450202

Miles, A.A., S.S. Misra and J.O. Irwin, 1938. The estimation of the bactericidal power of the blood. J. Hyg., 38: 732-749. PMID: 20475467

Naughton, P.J., G. Grant, R.J. Spencer, S. Bardocz and A. Pusztai, 1996. A rat model of infection by Salmonella typhimurium or Sal. enteritidis. J. Applied Microbiol., 81: 651-656. DOI: 10.1111/j.1365-2672.1996.tb03560.x
Naughton, P.J., L.L. Mikkelsen and B.B. Jensen, 2001. Effects of nondigestible oligosaccharides on Salmonella enterica serovar Typhimurium and nonpathogenic Escherichia coli in the pig small intestine in vitro. Applied Environ. Microbiol., 67: 3391-3395. PMID: 11472909

Ohl, M.E. and S.I. Miller, 2001. Salmonella: A model for bacterial pathogenesis. Annu. Rev. Med., 52: 259-274. PMID: 11160778

Poppe, C., 1999. Epidemiology of Salmonella enteric serovar Enteritidis. In: Salmonella enterica Serovar Enteritidis in Humans and Animals: Epidemiology, Pathogenesis and Control, Saeed, A.M., R.K. Gast, M.E. Potter and P.G. Wall (Eds.). WileyBlackwell, Ames, ISBN: 0813827078, pp: 3-18.

Quinn, P.J., M.E. Carter, B. Markey and G.R. Carter, 1993. Clinical Veterinary Microbiology. 9th Edn., Mosby, London, ISBN: 10: 0723417113, pp: 684.

Rahman, H., R. Reissbrodt and H. Tschape, 2000. Effect of norepinephrine on growth of Salmonella and its enterotoxin production. Indian J. Exp. Biol., 38: 285-286. PMID: 10927876

Santos, R.L., R.M. Tsolis, A.J. Baumler and L.G. Adams, 2003. Pathogenesis of Salmonella-induced enteritis. Braz. J. Med. Biol. Res., 36: 3-12. PMID: 12532221

Soliman, E.S., E. Taha, K.D. Infante, K. Laboy and M.A. Sobieh et al., 2009. Stressors influence on Salmonella enterica serovar enteritidis colonization in broilers. Am. J. Anim. Vet. Sci., 4: 42-48. DOI: $10.3844 / .2009 .42 .48$

Tobey, N., W. Heizer, R. Yeh, T.I. Huang and C. Hoffner, 1985. Human intestinal brush border peptidases. Gastroenterology, 88: 913-926. PMID: 3882510

Wehkamp, J., J. Harder, K. Wehkamp, B.W. Meissner and M. Schlee et al., 2004. NF-אB-and AP1 mediated induction of human beta defensin-2 in intestinal epithelial cells by Escherichia coli Nissle 1917: A novel effect of a probiotic bacterium. Infect. Immun., 72: 5750-5758. DOI: 10.1128/IAI.72.10.5750-5758.2004

Worton, K.J., D.C.A. Candy, T.S. Wallis, G.J. Clarke and M.P. Osborne et al., 1989. Studies on early association of Salmonella typhimurium with intestinal mucosa in vivo and in vitro: Relationship to virulence. J. Med. Microbiol., 29: 283-294. DOI: 10.1099/00222615-29-4-283

YashRoy, R., 2007. Mechanism of infection of a human isolates Salmonella (3,10:r:-) in chicken ileum: Ultrastructural study. Indian J. Med. Res., 126: 558-566. PMID: 18219084 